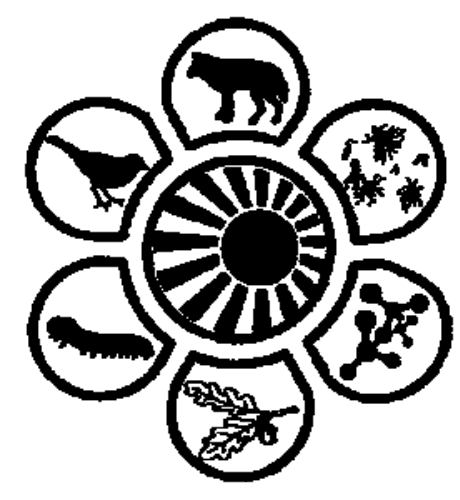

Вісник Дніпропетровського університету. Біологія, екологія.

Vìsnik Dnìpropetrovs'kogo unìversitetu. Serîa Bìologîâ, ekologiâ

Visnyk of Dnipropetrovsk University. Biology, ecology.

Vìsn. Dnìpropetr. Unìv. Ser. Bìol. Ekol. 2016. 24(2), 495-500.

doi: $10.15421 / 011667$

ISSN 2310-0842 print

ISSN 2312-301X online

www.ecology.dp.ua

UDC 582.394

\title{
Synopsis of the genus Anopteris \\ (Pteridophyta, Pteridaceae)
}

\author{
A.V. Vaganov ${ }^{1}$, A.I. Shmakov ${ }^{1}$, N. Friesen ${ }^{2}$

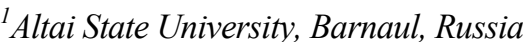 \\ ${ }^{2}$ University of Osnabrück, Osnabrück, Germany
}

In the article a synopsis of the genus Anopteris (Prantl) Diels is given. The synopsis of Anopteris includes three species. For each species, the Latin name, basionym, nomenclatural citation, synonyms, information on locus classicus, type, habitat, and distribution are given. An original key for identification of the species of Anopteris is also prepared. The localities of occurrence of each species are precisely given.

Keywords: Anopteris; synopsis; key; identification

\section{Конспект рода Anopteris (Pteridophyta, Pteridaceae)}

\author{
А.В. Ваганов ${ }^{1}$, А.И. Шмаков ${ }^{1}$, Н. Фризен ${ }^{2}$ \\ ${ }^{1}$ Алтайский государственный университет, Барнаул, Россия \\ ${ }^{2}$ Оснабрюкский университет, Оснабрюк, Германия
}

Приведен конспект рода Anopteris (Prantl) Diels. Конспект включает три вида. Для каждого из них приводится латинское название, базионим, номенклатурная цитата, синонимы, откуда описан, информация о типе, данные о местообитании, распространение по странам с цитированием мест сбора и общее распространение. Для определения всех видов рода Anopteris составлен оригинальный ключ, указаны подробные места произрастания.

Ключевые слова: Anopteris; конспект; ключ; определение

Altai State University, South-Siberian Botanical Garden, Lenin Ave., 61, Barnaul, 656049, Russia Алтайский государственный университет, пр. Ленина, 61, Барнаул, 656049, Россия E-mail:vaganovvvav@mail.ru

University of Osnabrück, Botanical Garden, Albrechtstrasse, 29, Osnabrück, D-49076, Germany

Оснабрюкский университет, ботанический сад, Альбрехтитрассе, 29, Оснабрюк, D-49076, Германия

Visn. Dnipropetr. Univ. Ser. Biol. Ekol. 2016. 24(2) 


\section{Introduction}

Representatives of Anopteris occur exclusively on the islands of Central America where they grow predominantly in rock crevices and on calcareous slopes at the elevation of $800 \mathrm{~m}$ a. s. 1. or below.

Position of Anopteris (Prantl) Diels in the present-day pteridological systems is not finally determined and the genus is being treated a member of the heterogenous Pteridaceae E.D.M. Kirchn. (Smith et al., 2006; Schuettpelz et al., 2007; Christenhusz et al., 2011). Our studies of general morphology, anatomy, and spore characters (Kuznetzov et al., 2013, 2014; Vaganov et al., 2014) demonstrate that Anopteris is closest to some representatives of Cryptogrammoideae S. Linds. of Pteridaceae. As a result of taxonomic revision (Vaganov et al., 2010), Anopteris has been found to include three species: A. hexagona (L.) C. Chr., A. strictum (Kunze) A. Vaganov et Shmakov, A. intermedia (Morton) A. Vaganov et Shmakov.

\section{Materials and methods}

A synopsis is based on the revision of herbarium material of B, P, PE, and LE as well as relevant literature.

\section{Results}

Anopteris (Prantl) Diels, 1902, in Engler et Prantl, Nat. Pflanzenfam. 1(4): 288; Proctor, 1985, Ferns Jamaica: 264. Cryptogramme sect. Anopteris Prantl, 1882, in Engler, Bot. Jahrb. 3: 414.

Plants 15-60 cm high. Sterile apex of fertile segment usually well-developed, rather big, distantly lobed or serrate at margin. All stipes opposite. Ultimate segments of sterile frond entire, margin serrate; frond 2-5-pinnate. $\mathrm{n}=58,2 \mathrm{n}=116$.

Typus: "Anopteris hexagona (L.) C. Chr., based on Adiantum hexagonum L. of the West Indies" (Proctor, 1985).

Three species on the islands of Central America at the elevation of 150 to $800 \mathrm{~m}$ a. s. 1 .

\section{Key for species of Anopteris}

1. Frond lax, spreading, 3-5-pinnatisect. Ultimate segments of sterile fronds on winged stipes, scarious, narrow, pinnately divided to the middle or deeper. Rachis with scales laxly arranged throughout or in the proximal half

- Fronds erect, laminas leathery, 2-4-pinnatisect. Ultimate segments of sterile fronds on wingless stipes, coriaceous, undivided, broadly ovate to ovate-lanceolate, margin dentate to crenate. Scales developed at the base of rachis only ......

2. Rachis $1-1.5$ times as long as the lamina. Laminas of sterile fronds 2-3-pinnatisect. Ultimate segments of fertile fronds 4-10 times longer than wide. Sterile apex of fertile segment well-developed, big ..... 1. A. hexagona

- Rachis 1.5-2 times as long as the lamina. Laminas of sterile fronds 3-4-pinnatisect. Ultimate segments of fertile fronds 2-6 times longer than wide. Sterile apex of fertile segment poorly developed, dentate at margin (often in case of incomplete frond dimorphism), or absent (usually in case of complete frond dimorphism) 3. A. intermedia
1. A. hexagona (L.) C. Chr., 1905, Ind. Fil.: 59; Proctor, 1985, Ferns Jamaica: 264. - Pteris heterophylla L. 1759, Syst. Nat. ed. 10, 2: 1322. - Allosorus heterophyllus (L.) Bernh. 1806, Neues Journ. Bot. (Schrad.) 1 (2): 36. - Cryptogramma heterophylla (L.) Prantl, 1882, in Engl. Bot. Jahrb. 3: 414. - Onychium heterophyllum (L.) Kuhn ex Krug, 1897, in Engl. Bot. Jahrb. 24: 98. - Anopteris heterophylla (L.) Diels, 1902, in Engler and Prantl, Nat. Pflanzenfam. 1 (4): 289. - Pteris hexagona (L.) Proctor, 1953, in Bull. Inst. Jamaica, Sci. Ser. 5: 53. - Anopteris hexagona var. simplicior Morton, 1962, Amer. Fern Journ. 52, 4: 148; Proctor, 1985, Ferns of Jamaica: 266. - Anopteris hexagona var. hexagona Proctor, 1985, Ferns of Jamaica : 266. Adiantum hexagonum L. 1753, Sp. Pl. 2: 1097.

Described from Haiti isl.

Typus: "Petiver, Pter. Amer. t. 10, fig. 2, redrawn (one frond only) from Plumier, Tr. Foug. t. 37, in turn based on a plant from near Anses a Pitre, district of Leogane, Haiti" (Proctor, 1985, Fig. 1).

Calcareous slopes, rock crevices.

Distrib.: Jamaica isl.: Hort Kew, 1857; West Indies №392; Jamaica, Ugly River, W. Harris, №7425, 8.10.'98; H. Kew "Lph", 1865; Menderille, Jamaica, №84; Jamaica, E herba horti Botan. Jamaica, a. 1890, mis Duss.; Jamaica, ..., ..., №4925, ... Angnstin 1858, Antill, 4.0, H. Bersh, 54; Kew, Ankauf von Renate Lange, 4.10.1960, 1887, №79; Jamaica, №77; Jamaique, Deep woods, Hollymount, 3.7.16, №436; Mone. Jamaique, april 1920, ...; Jamaica, Bath fountain, Mar 5 1900, №284, altitude 400ft; Jamaica, №717, Herb. Brandford; Jamaica, Hartford and adjoining properties, near Priestman's River, altitude 75-300 meters, wel rocks in ravine, №2547, W.R. Maxon, June 9 1904; On wel "ohrded" rocks, №763, W.R. Maxon, April 8, 1903; Jamaica, Consul Ebbeke, Prof. Binthenan comm., 1885; Jamaique, Aug 6 Lewis, 2 1860; dela Jamaique, acritura das wartz, 1830; Fern Gully, Island of Jamaica, West Indies, March 191897 , J.R. Churchill; Jamaica, West Indies, March 1858, №392; Jamaica, Spur of Crow Mounntains opposite Mill Bank, Portland, in deep forest, altitude 450-625 meters, №9370, W.R. Maxon, June 18, 1926; Jamaica, West Indies, №392; Jamaica, Dec 1890, A.S. Hitchcock s.n.; Jamaica, March 19, 1897, J.R. Churchill s.n.; Jamaica, 600 f, Mar 5, 1900, Willard N. Clute 284; Jamaica, 1886, JP s.n.; Jamaica, March 1895, B.D. Gilbert s.n.; Jamaica, Jan 1, 1929, C.R. Orcutt 7697; Jamaica, July 31, 1932, T.S. Johnson s.n.; Jamaica, July 1891, Anonymous s.n.; Jamaica, Anonymous s.n.; Jamaica Portland, 1500 f, 27 July 1963, M.R. Crosby, H.A. Hespenheide a. W.R. Anderson 970; Jamaica Portland, 2500 f, January 8-10, 1951, George R. Proctor 5212; Jamaica Portland, 450-625 m, June 18, 1926, William R. Maxon 9370; Jamaica Portland, April 16, 1950, George R. Proctor 4277; Jamaica Trelawny, March 6, 1950, George R. Proctor 4117; Jamaica Hanover, 1500-1700 f, 11 April 1955, George R. Proctor 10023; Jamaica Trelawny, December 30, 1949, George R. Proctor 3827; Mt. Ridgway Road. Windsor, Parish of Trelawny; altitude 100 to 150 meters. №1557. Gerrit S. Miller, Coll. April 9, 1931; Lemestone rocks, in thin shade. Fern Gully, St. Ann; altitude 200-300 meters. №10375. William R. Maxon, Coll.; Haiti isl.: Haiti, Vicinity of Mi..ion, Fonds Varettes, altitude about 1000 meters, Dump shaded ..., №3777, Leonard, 27.4.1922; Haiti, Vicinity of Mi..ion, Fonds Varettes, altitude about 1000 meters, Rid "iliclad", Leonard, 
№4013, 27.4.1922; Haiti, Damp rocks, №3761, Leonard, 27.4.1922; Dominican Republic Pedernales, 3900 f, $18^{\circ} 09^{\prime} \mathrm{N}$ 071 $38^{\prime}$ 'W, September 18, 1981, T. Zanoni, M. Mejia 16719; Haiti, 1900 m, $18^{\circ} 20^{\prime} \mathrm{N} 072^{\circ} 16^{\prime} \mathrm{W}$, Nov 21, 1982, T. Zanoni, M. Mejia a. J. Pimentel 24546; Vicinity of Mission, Fonds Varettes; altitude about 1000 meters and above. №4011. E.C. Leonard, Collector. April 17-May 4, 1920; Cuba: Cuba,
Santjago de la Vegas, 5.1905. ...F. Baker; Cuba Guantanamo, 330 m, 17 Jun 1997, F.S. Axelrod, J. Ackerman, M. Diaz a. B. Zabala 10336; Bermuda isl.: Bermuda, Mr. Moseley, Challenger Expedition, Recd. Aug. 1873) (Pl. fil. t 34, slow. h. it. 34, Habitat in "antillir", 4; Bermuda, March 1877, G. Browngoode 2001; Bermuda, 1880, Farlow s.n. - Gen. distr.: Central America, Bermuda isl.

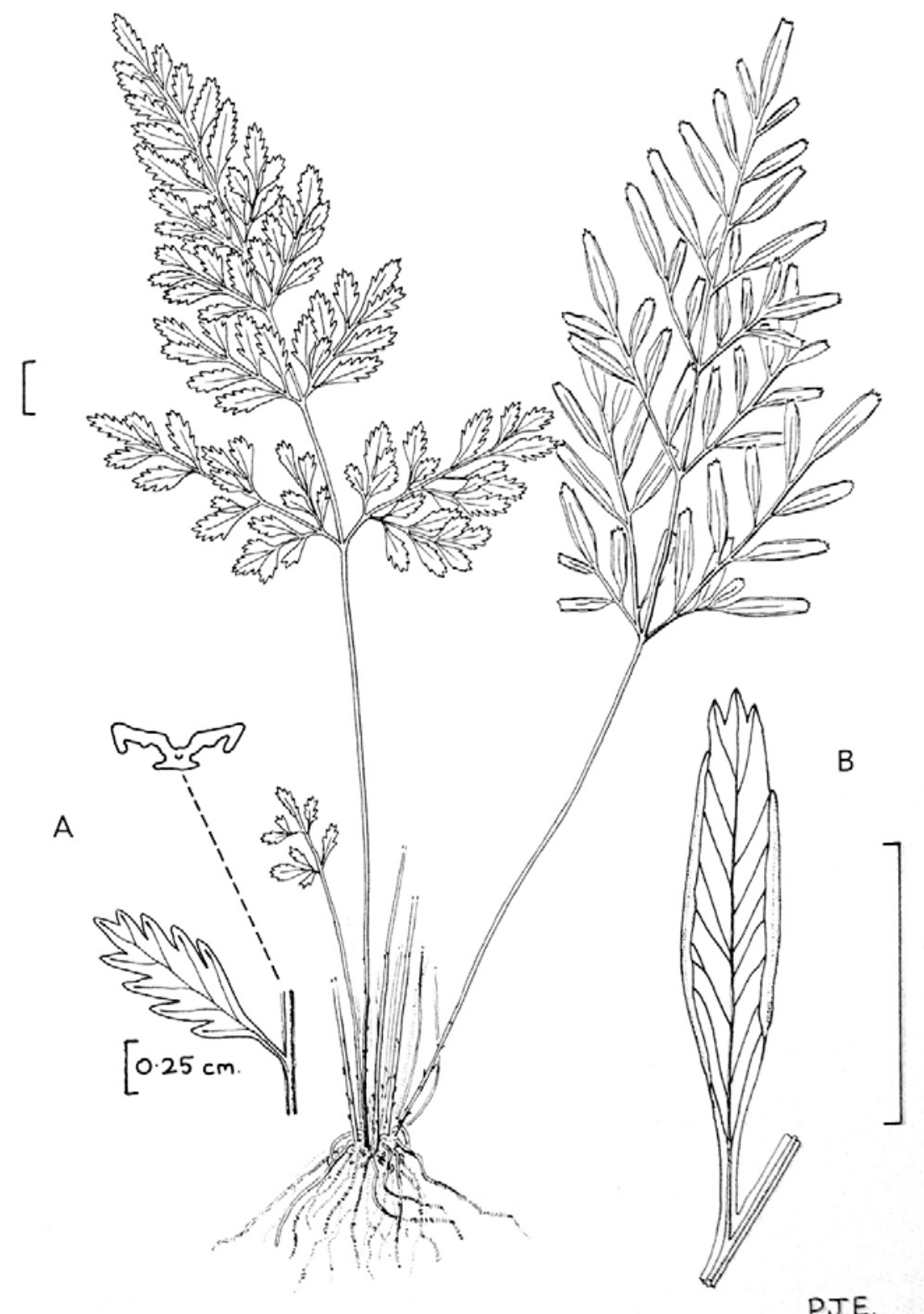

Fig. 1. Type of Anopteris hexagona (L.) C. Chr. (f. 68. From Proctor, 1985)

2. A. stricta (Kunze) A. Vaganov et Shmakov, 2010, Turczaninowia, T. 13 (1): 9; Kummerle, 1930, Amer. Fern Journ. 20 (4): 137. - Onychium multifidum Fee, 1857, Mem. Foug. 8: 74. - Anopteris hexagona subsp. multifida (Fee) Morton, 1957, Bull. Jard. Bot. Etat 27: 584. - Onychium strictum Kunze. 1848, in Schkuhr's Fil. Suppl. 2 ; 11.

Described from Cuba isl.

Isotypus: 'Cuba, Prov. de Sant Yago, Mt. Liban., 6000', J. Linden, №1870, May 1844” (K, photo LE!, Fig. 2).

Rock crevices, slopes.

Distrib.: Haiti isl.: Haiti, in moutibas ad "Meru gouns pospe Pay. in praerup ....", 1891, "Picardu", №199; Haiti, pr Kze is del ... Payaur, 100 m, ..., 1891, №1894; Hispaniola,
Civ. Haiti, Maruf de la Holle, western group, Camp Perrin northern slope of M. Vandervelde, in Jardins Crutard, limestone gerill, chady plare, ca 800 m, 2.12.1921, E.L. Ekman, №5231; Ind. Occ. Ins. Hispaniola, civ. Haiti, Dep. du Sud. Morne de la Hofa in declier sopt.-orient in sylvis montain, c. 800 m.s.m., in supibus, 2.6.1917, E.L. Ekman, №177; Hispaniola, Civ. Haiti, Manif de la Hotte, dr. Morne Rochelois, Miragoane, limestone irag at Kuatre-Cheming, 1000 m, 17.7.1927, E.L. Ekman, №8607; Haiti, "Rosine Guitte de ... pr. Peyan, Hauteurs de Miragoane, Yan 1891, Pirarda, №199; Hispaniola, Civ. Haiti, Manif de la Selle, gr. Mote des Gommissaires, Grand-Gosier at Sourde - Rouille shaded limestone, 820 m, 8.9.1926, E.L. Ekman, №6836; Puerto 
Rico isl.: Puerto Rico, Rio Abajo state forest, Along hwy, 621 , near and of asphalt road, $18^{\circ} 19^{\prime} \mathrm{N}, 66^{\circ} 40^{\prime} \mathrm{W}$, elev. 360 390 m, Eplpetric, 2 Aprill 1985, Thomas B., Croat 60885; Areribo, in sybvis, 28.2.1887, det. M. Kuhn, №6331; Sto. Domingo austr. prope Barahona, 1912, Padre Miguel Fuertes, №1395b; Sto. Domingo austr. prope Barahoma, 4.1912, Filipines 850 ..., Padre Miguel Fuertes, №1519; "Juria de Lares in huruides rybr frein area Yuajataca adrupes", 16.2.1887, Kuhr, №6250; Prope Arecibr in sylva prunaeva ad rupes calcareas, 28.2.1887, Kuhn, №6331; Prope Uluadr in sylva prunaeva ad rupes calcareas, 23.3.1887, №6580; Sierra de Lares in humides sylvae prunaevae circa Guajataca ad rupes, rara, 16.2.1887, №6250; Areribo, in sylvis, 28.2.1887, M. Kuhn, 6331; Sierra de Lares, in sylva ad Guajataca, 16.2.1887, M. Kuhn, №6250; Flora porforiceutis, Sierra de Lares, in humides sylvac primacoac, 16.2.1887, Sinteuis; Utuadr, in sylva primaelva, 23.3.1887, M. Kuhn, №6580; Puerto Rico, 360-390 m, $18^{\circ} 19^{\prime} \mathrm{N} 066^{\circ} 40^{\prime} \mathrm{W}, 2$ April 1985, Thomas B. Croat 60885; Puerto Rico, 150-200 m, 23 Jun 1993, F. Axelrod a. L. Sanchez 6508; Puerto Rico, 300 m, 18²0’N 066²4'W, 18 Oct 1995, F.S. Axelrod 9269; Municipio Utuado, Rio Abajo
State Forest, ca. $1.6 \mathrm{~km}$ WSW of Campamento Crozier, ca. 1-1.5 mi. S along trail from Rt. 621. Lemistone sink holes in rich mixed forest. Coll. Z.R. Wang, A.M. Evans, G.R. Proctor and R. Rivero. №91069. Apr 5, 1991. Cuba: Cuba, "Lans del Juqucy", 700 m, 0,7 m alt. "su ... hibus", №4931, Mart 1889; Cuba, C. Wright, 1865, no.814; Cuba, Loma del Juqucy, 700 m, 0,3 m alt. Juter saxa", Marz 1889, №4917; Cuba, Yateres Oricute, Joscphina $575 \mathrm{~m}$, Maxon, 4.07; Cuba Oriental, prope vllam Monte Verde dietam, Jan.-Jul. 1859; C. Wright, №858; Cuba, Linden, 1843-4, №1840; S’ Yago de Cuba, 1844, Linden, №1870; Cuba, Loma del jaqucy 600 m, 0.3 alt. "leraboribus mortus", Mart 1889, №4931; Cuba, C. 98, Kze, №814, C. Wright, 1865; Cuba, L.M. Underwood and F.S. Earle, March 1903, №954; Cuba, L.M. Underwood and F.S. Earle, March 1903, №1053; Cuba, Yateras Oriente Josephina 575 m., 4.1907, Maxon; La Perla, 600 m, Jullie 1919, №2471; Slopes and summit of El Yunque, near Baracoa, Altitude 1000 to 2000 feet, C.L. Pollard, W. Palmer, January 30-31, 1902, №116; Josephina, north of Jaguey, Yateras, Oriente, altitude about 575 meters, Cuba, Yateras Oriente Josephina 575 m, 4.1907, Maxon, №4110. - Gen. distr.: Central America.

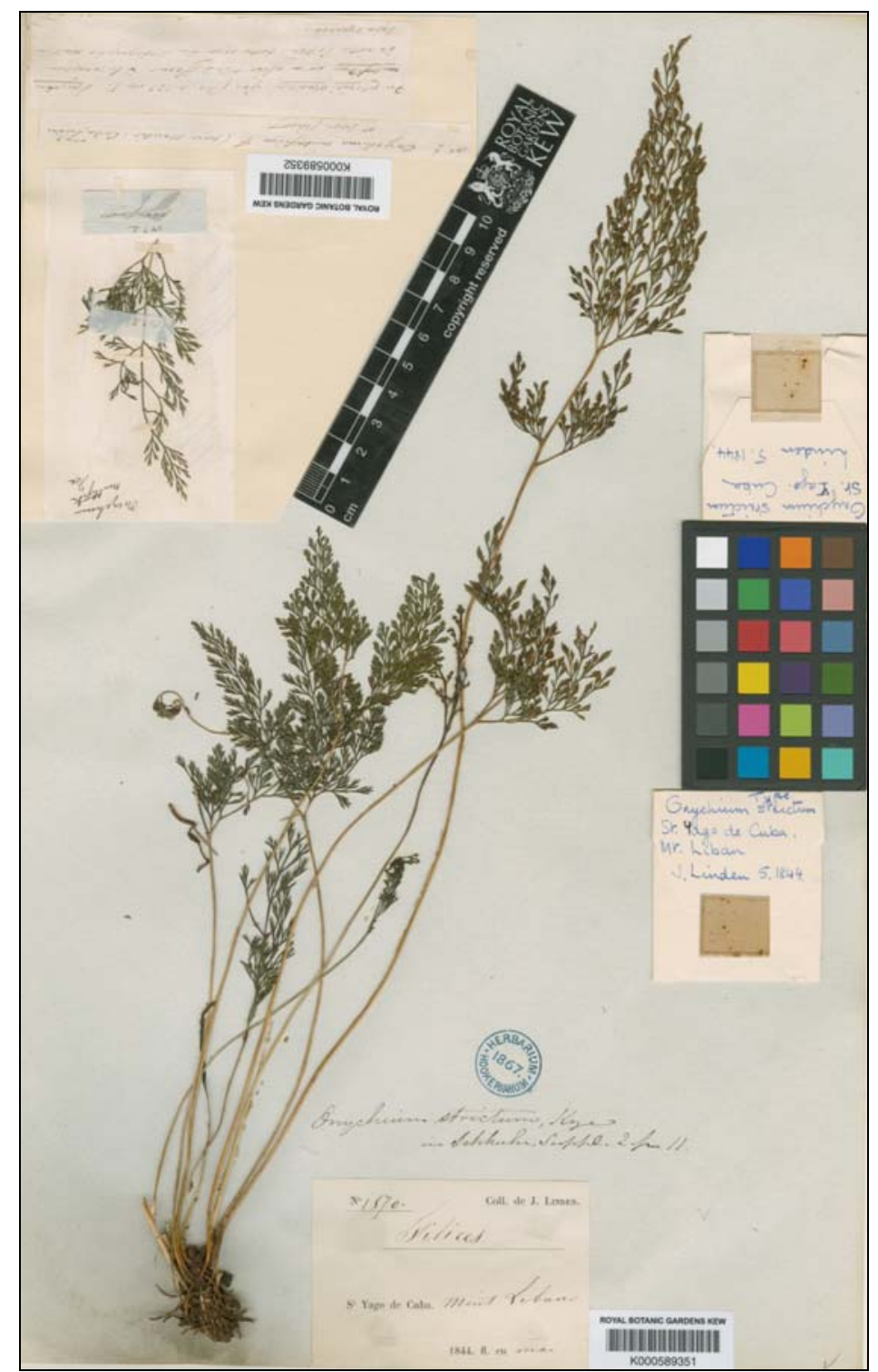

Fig. 2. Isotype of Anopteris stricta (Kunze) A. Vaganov et Shmakov (K) 


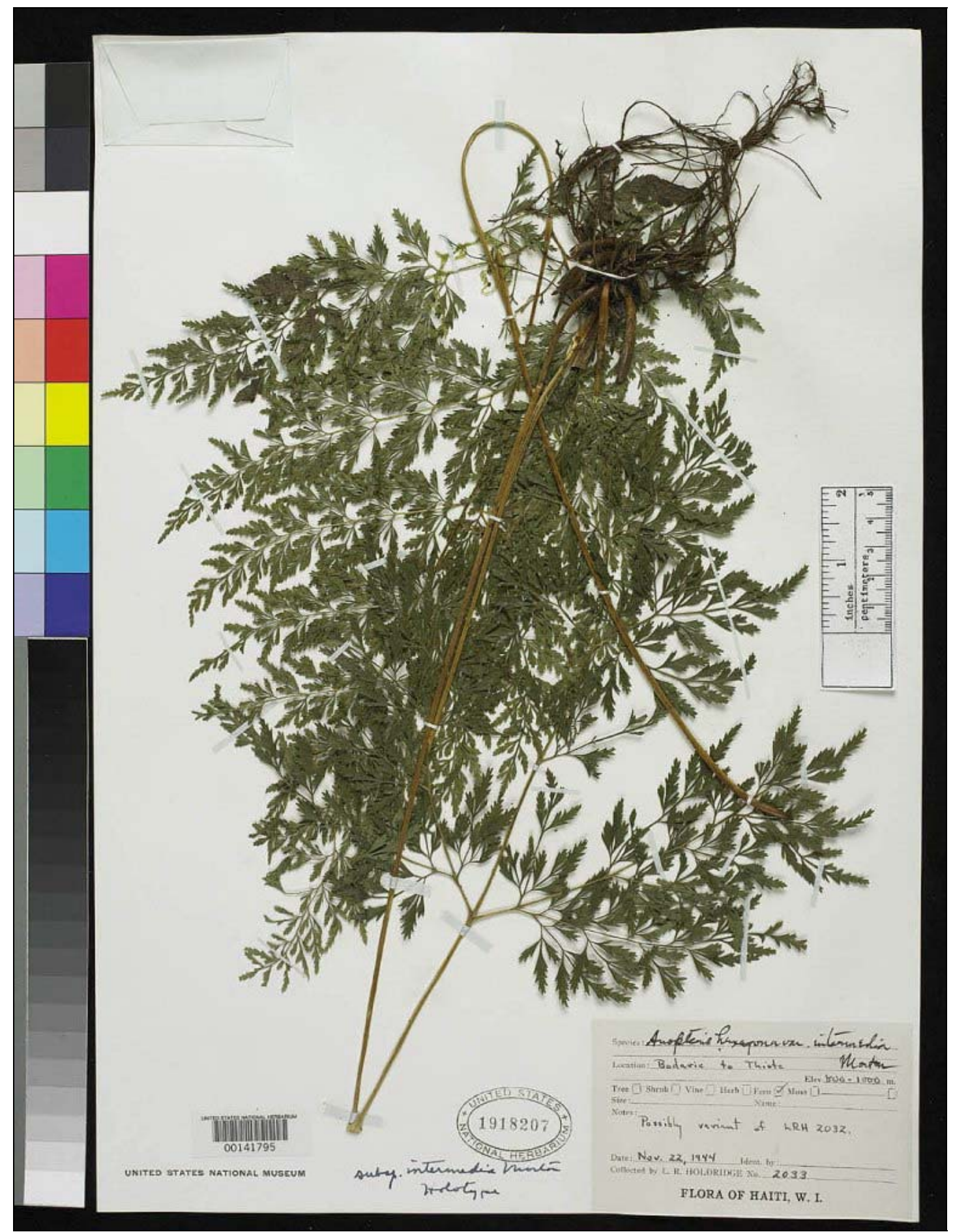

Fig. 3. Holotype of Anopteris intermedia (Morton) A. Vaganov et Shmakov (US)

3. A. intermedia (Morton) A. Vaganov et. Shmakov, 2010,

Turczaninowia, T. 13 (1): 10. - Anopteris hexagona subsp. intermedia Morton, 1957, Bull. Jard. Bot. Etat 27: 583.

Holotypus: «Haiti, Bodarie to Thiete, Hispaniola Island, 800-1000 m., 22 Nov 1944, L.R. Holdridge» (US, Fig. 3).

Rock crevices, slopes.

Distrib.: Haiti isl.: Heiti, "Tlanrs du Mirne trnshant 1700 m, Borts du chemin", 8 Aout, "Dira..."№745; Haiti, Telice, Morne tranchaut, schattige Schlucht, $1600 \mathrm{~m}, 50-70$.. hoch, №1961; Haiti, Picarda, In dechisibus montis Morne Tranchaut ad oriam, alt. 1600-1700 m, 8.8.1891, №745; Prov. Barahona ad Las Filipinas $830 \mathrm{~m}$ alt., Padre Miguel Fuertes, 1912, №1519. - Gen. distr.: Central America.

\section{References}

Christenhusz, M.J.M., Zhang, X.-C., Schneider, H., 2011. A linear sequence of extant families and genera of lycophytes and ferns. Phytotaxa 19, 7-54.

Diels, L., 1902. Polypodiaceae. In: Engler, A., Plantl, K. (eds). Die naturlichen Pflanzenfamilien 1, 4. W. Engelmann, Leipzig.

Kummerle, J.B., 1930. Has the Genus Onychium any representative in South America. Am. Fern J. 20(4), 129-138.

Kuznetsov, A.A., Vaganov, A.V., Gureyeva, I.I., Shmakov, A.I., 2013. Morfologija spor paporotnikov semejstva Cryptogrammaceae i blizkih $\mathrm{k}$ nemu rodov [Spore morphology of 
the Cryptogrammaceae ferns and allied genera]. Barnaul University Press, Barnaul (in Russian).

Kuznetzov, A.A., Vaganov, A.V., Skapcov, M.V., Erst, A.S., 2014. A comparative study of spore morphology of some Pteridoideae subfamily genera. Biosciences Biotechnology Research Asia 11, 17-25.

Morton, C.V., 1962. Taxonomic notes on ferns, 3. Am. Fern J. $52(4), 148-150$.

Morton, C.V., 1957. The fern genus Anopteris. Du Jardin Botanique National de Belgique 27, 579-584.

Prantl, K., 1882. Die Farngattungen Cryptogramme und Pellaea. Separal-Abdruck aus Botanischen Jahrbuchern 3. Band. 5. Leipzig. P. 403-430.

Proctor, G.R., 1985. Subfamily Pteridoideae. In: Ferns of Jamaica. A guide to the Pteridophytes. British Museum (Natural History). P. 264-283.

Schuettpelz, E., Schneider, H., Huiet, L., Windham, M.D., Pryer, K.M., 2007. A molecular phylogeny of the fern fami- ly Pteridaceae: Assessing overall relationships and the affinities of previously unsampled genera. Mol. Phylogenet. Evol. 44, 1172-1185.

Smith, A.R., Kathleen, M.P., Schuettpelz, E., Korall, P., Schneider, H., Wolf, P.G., 2006. A classification for extant ferns. Taxon 55(3), 705-731.

Vaganov, A.V., Shalimov, A.P., Shaulo, D.N., 2014. Morfologija spor nekotoryh predstavitelej podsemejstva Pteridoideae semejstva Pteridaceae [Spore morphology of some representatives of Pteridaceae subfam. Pteridoideae]. Plant Life of Asian Russia 14, 29-36 (in Russian).

Vaganov, A.V., Kuznetsov, A.A., Shmakov, A.I., 2010. Taksonomicheskaja revizija roda Anopteris (Prantl) Diels (Cryptogrammaceae) [Taxonomic revision of the genus Anopteris (Prantl) Diels (Cryptogrammaceae)]. Turczaninowia 13(1), 5-13 (in Russian).

Надійила до редколегї 25.09.2016 